\title{
APLICAÇÃO PROGRESSIVA WEB DE CÓDIGO ABERTO COMO MECANISMO PARA REPRESENTAÇÃO SIMPLES DE TERRENO.
}

\author{
F.A. Silva ${ }^{1,}{ }^{*}$ C.L. Tavares $^{1,2}$; A.R. Neves ${ }^{1}$ \\ 1 - Faculdade de Tecnologia de Jacareí - Professor Francisco de Moura \\ Av. Faria Lima, 155 - Jardim Santa Maria, Jacareí/SP, \\ CEP.: 12328-070, Brasil. \\ Telefone: (12) 3953-7926 \\ 2 - Serviço Nacional de Aprendizagem Industrial de Santa Catarina \\ Rod. SC-401, 3730 - Saco Grande, Florianópolis/SC, \\ CEP.: 88032-005, Brasil. \\ Telefone: (48) 3239-5800 \\ *azevedo.fernando.geo@gmail.com
}

\begin{abstract}
RESUMO: A tecnologia geográfica já é fato na sociedade, por isso o conhecimento de alguns fundamentos relacionados ao tema é relevante. Nesse cenário, o objetivo é o desenvolvimento inicial de uma Aplicação Progressiva Web (PWA) que visa ajudar os interessados em geotecnologias a compreenderem, de maneira simplificada, os fundamentos da geração de um Modelo de Terreno através de sua representação por Rede Triangular Irregular (TIN). O uso em futuras versões melhoradas dessa tecnologia possibilitaria que não apenas entusiastas mas também alunos e professores visualizassem parte da topografia do município, além da oportunidade de conhecimento da altimetria local por meio da análise do caminho percorrido. Desse modo, a utilização do mecanismo como uma ferramenta pedagógica auxiliar poderia ser viável.
\end{abstract}

PALAVRAS-CHAVE: Modelo de Terreno; TIN; PWA; geotecnologias.

ABSTRACT: The geographic technology is already a fact in society, so the knowledge of some fundamentals related to the theme is relevant. In this scenario, the goal is the initial development of a Progressive Web Application (PWA) which aims at helping the ones interested in geotechnologies understand, in a simplified way, the foundations of the generation of a Terrain Model through its representation by Triangulated Irregular Network (TIN). The use in future of enhanced versions of this technology would allow not only enthusiasts but also students and Professors to visualize part of the municipality topography, besides the possibility of knowing the local altimetric through the analysis of the covered location pathway. In that way, the utilization of the mechanism as an auxiliar pedagogical tool could be practicable.

KEYWORDS: Terrain Model; TIN; PWA; geo-technologies.

\section{INTRODUÇÃO}

Para Moran (1997) a internet pode ser considerada um facilitador para a motivação dos alunos, sendo que essa motivação pode aumentar se o professor a faz em um clima de confiança, de abertura, de cordialidade com os discentes. Corroborando com ideia anterior, Jacobi (2003) diz que em tempos em que a informação assume um papel cada vez mais substancial: o ciberespaço, 
multimídia, internet e a educação para a cidadania representam a possibilidade de instigar e sensibilizar as pessoas para transformar as diversas formas de participação na defesa da qualidade de vida.

Em se tratando de geotecnologias, uma didática simples que possa ser compreendida de maneira mais fácil pelos interessados com pouco conhecimento neste tema tende a ser importante. Por isso, novas aplicações web mobile podem ser criadas como ferramenta auxiliar para curiosos da matéria, além da possibilidade de servir a alunos e professores. Assim, um aplicativo multiplataforma que explique, de modo simplificado, como a geração de modelos de elevação acontece pode ajudar no entendimento deste assunto. Para tanto, as linguagens de programação são parte integrante do desenvolvimento.

De acordo com Rossum e Drake Jr. (2011), Python foi criado em 1990 pelo próprio Rossum na Holanda com auxílio de outros colaboradores, sendo esta linguagem de programação sucessora de uma anterior chamada ABC. Ela possui, como uma de suas características, bibliotecas que são capazes de auxiliar a análise estatística e geográfica, ou seja, estudos geoestatísticos.

Os esforços do engenheiro de minas Daniel G. Krige e as publicações posteriores com base nestes trabalhos, como as de G. Matheron na década de 60 foram muito importantes para geoestatística, sendo que esta última é intimamente relacionada com o estudo de variáveis regionalizadas que, por consequência, possuem condicionamento espacial (LANDIM, 2006).

O Modelo Digital de Terreno ou MDT (do inglês: Digital Terrain Model - DTM) pode ser obtido através de interpolações matemáticas sobre dados espaciais. De acordo com Felgueiras (1999), tal tipo de modelagem representa o comportamento de um fenômeno que ocorre em uma região da superfície terrestre e os dados de MDTs são de fundamental importância em aplicações de geoprocessamento desenvolvidas no ambiente de um Sistema de Informações Geográficas (SIG).

Segundo Egg (2012), outras denominações para conceituar os modelos que representam tridimensionalmente os diferentes fenômenos que ocorrem no espaço geográfico existem, como o Modelo Digital de Elevação (MDE) e o Modelo Digital de Superfície (MDS). O serviço de Pesquisa Geológica dos Estados Unidos (do inglês: United States Geological Survey - USGS) passa a seguinte informação sobre os modelos citados que são disponibilizados pelo serviço, considerando que suas modelagens foram feitas tendo como base as imagens SRTM (Shuttle Radar Topography Mission):

- O MDE (do inglês: Digital Elevation Model - DEM) é uma representação do terreno (terra nua) com elevações em intervalos regularmente espaçados (UNITED STATES GEOLOGICAL SURVEY, 2019);

- O MDS (do inglês: Digital Surface Model - DSM) também contém elevações em intervalos regularmente espaçados, contudo as elevações representam a primeira superfície refletida detectada pelo sensor, assim, esses primeiros retornos podem ser refletidos pelo solo nu ou por características da superfície como árvores e estruturas (UNITED STATES GEOLOGICAL SURVEY, 2019).

Um MDE pode ser representado como uma imagem matricial, onde cada valor pixel representa uma informação (no caso do DEM é a elevação), além disso, a aquisição dos dados para se fazer as modelagens podem ser por meio de técnicas como fotogrametria, levantamento topográfico e outras. (LI; ZHU; GOLD, 2005). De acordo com Teske, Giasson e Bagatini (2014), pode-se gerar dados diretamente do MDE, como: declividade, orientação das vertentes, entre outros.

De acordo com o United States Geological Survey (2018), a Shuttle Radar Topography Mission voou a bordo do ônibus espacial americano Endeavor de 11 a 22 de fevereiro do ano 2000, tendo como participantes de um projeto internacional a Agência Espacial dos Estados Unidos (do inglês: National Aeronautics and Space Administration - NASA) e a Agência Geoespacial de Inteligência dos Estados Unidos (do inglês: National Geospatial-Intelligence Agency - NGA). 


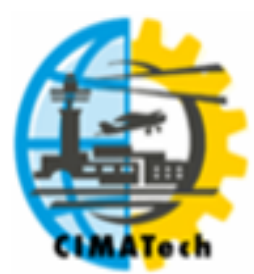

Ainda de acordo com o USGS, o objetivo foi a aquisição de dados de radar que foram usados para criar o primeiro conjunto de elevações de grande parte do planeta Terra, esta missão usou duas antenas de radar diferentes: uma antena ficou localizada a bordo do ônibus espacial e coletou um conjunto de dados, enquanto a outra estava no final de um mastro de 60 metros que se estendia do ônibus espacial e coletou o outro conjunto de dados. As diferenças entre os dois sinais permitiram o cálculo da elevação da superfície por meio de interferometria (UNITED STATES GEOLOGICAL SURVEY, 2018).

Segundo Bhargava, Bhargava e Tanwar (2013), a Rede Triangular Irregular (do inglês: TIN Triangulated Irregular Network), baseada em vetor, nada mais é do que um modo de representar uma superfície derivada de pontos de amostra espaçados irregularmente e recursos de linha de quebra. Desta forma, em conjunto com a localização do usuário, é possível gerar um gráfico em três dimensões (3D) e o caminho de altitude percorrido a partir da base de dados de modo dinâmico, de maneira que a partir de um DEM derivado de imagens SRTM é possível se fazer a representação vetorial do terreno em forma de TIN por meio de amostragem. Para isso, utilizou-se aqui ferramentas de geotecnologia para visualizações simples de elementos de geoprocessamento em uma Aplicação Progressiva Web (do inglês: Progressive Web Application - PWA).

Os PWAs são uma nova forma interessante de aplicativos modernos, aproveitando os recursos mais recentes da internet para oferecer uma experiência que combina os recursos de aplicativos móveis nativos com as vantagens da web (ALTER, 2017). Tais características fazem com que eles possam ser usados em computadores de mesa ou em tablets e smartphones, pois consomem recursos dos navegadores de cada dispositivo. Nesta conjuntura, o objetivo é o desenvolvimento inicial de uma Aplicação Progressiva Web que visa ajudar os interessados em geotecnologias a compreenderem, de maneira simplificada, os fundamentos da geração de um Modelo de Terreno através de sua representação por Rede Triangular Irregular.

\section{MATERIAIS E MÉTODOS}

A aplicação foi pensada para que os usuários pudessem realizar parte dos procedimentos sem necessitar de conexão com a internet. Por isso, trabalhou-se principalmente com as seguintes bibliotecas Javascript de código aberto: Leaflet para a produção dos mapas, pois é uma biblioteca enxuta e com boa documentação; Plotly que possibilita que os gráficos sejam visualizados; Turf que tem como cerne as funções geográficas, possibilitando o perfil altimétrico do caminho percorrido como produto, além de mostrar a lonjura entre as localizações.

Um outro produto é o gráfico em três dimensões que é feito dinamicamente com o auxílio da biblioteca Matplot do Python, porém, neste caso, a visualização requer conexão com a rede mundial de computadores.

\subsection{Descrição do Local}

O local geográfico inicial escolhido para a retirada das amostras digitais por meio de imagem SRTM foi o município de Jacareí, localizado na Bacia do Rio Paraíba do Sul no Estado de São Paulo - Brasil. Faz divisa com as cidades de São José dos Campos, Santa Branca, Jambeiro, Guararema, Santa Isabel e Igaratá. Além disso, conta com uma população estimada de mais de 230 mil pessoas segundo o Instituto Brasileiro de Geografia e Estatística (IBGE, 2018).

A Figura 1 mostra o mapa de altimetria de Jacareí com o sistema de referência WGS 84 e com a legenda de altitude dada em metros. Os dados de imagem SRTM são advindos do Instituto Nacional de Pesquisas Espaciais (INPE) mediante o site TOPODATA MAPA ÍNDICE (2019). Já os limites apresentados são advindos da malha digital do Instituto Brasileiro de Geografia e Estatística do ano de 2015 (IBGE, 2016), excluindo o dado de delimitação da bacia do Rio Paraíba 


\section{ISSN $2447-5378$}

do Sul obtido pelo banco de dados da Agência Nacional de Águas (ANA) e modificado por meio de uma operação espacial no software Qgis para se obter apenas sua porção paulista. De acordo com ANA (2018), a divisão em sub-bacias foi feita em 1972 pelo Departamento Nacional de Águas e Energia Elétrica - DNAEE.

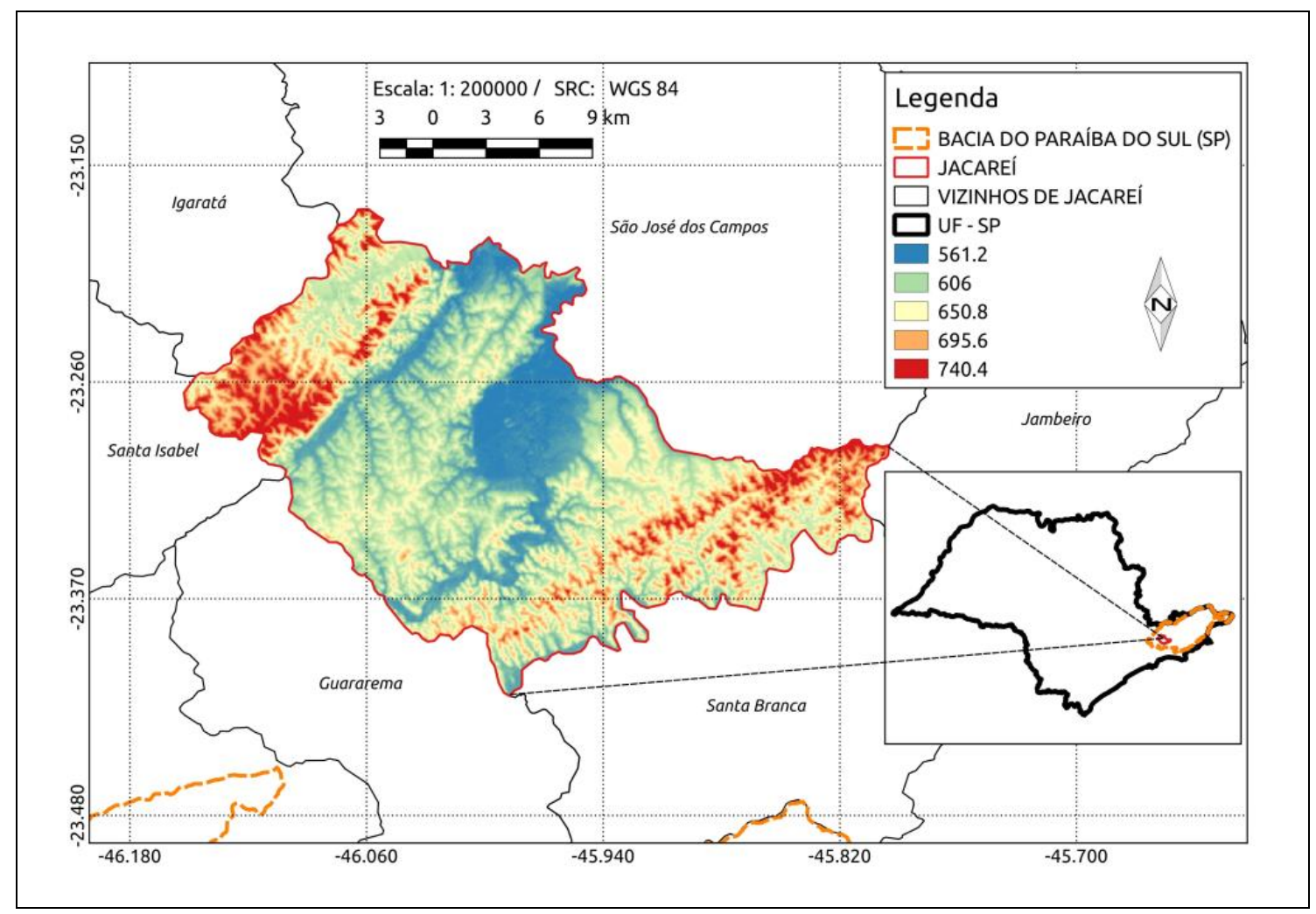

Figura 1. Mapa de Altitude de Jacareí e sua delimitação (AUTOR, 2019).

\subsection{Método de Amostragem}

Utilizou-se amostragem aleatória simples para se obter os pontos necessários para a representação do terreno por meio do TIN. Isto ocorreu por conta de sua adequação com a especificidade da base de dados (DUMITRU; PLOPEANU; BADEA, 2013).

\subsection{Obtenção das Amostras}

Os dados foram obtidos a partir de imagem com dados de altitude STRM do site TOPODATA MAPA ÍNDICE (2019) do INPE e o SIG utilizado para retirada das amostras foi o Qgis em sua versão 2.18. Em um primeiro momento foram retirados mais de 70 mil pontos aleatórios da imagem com distância mínima de 50 metros entre cada ponto a partir das delimitações da cidade para não ocorrer demasiado agrupamento de pontos em distâncias muito próximas a cada coordenada, contando com um aumento de limite de suas fronteiras em 200 metros que pode ser observado na Figura 2 - o mapa mostra à esquerda o zoom de uma área na divisa com São José dos Campos e à direita os todos os pontos amostrados em Jacareí. Os limites citados são advindos da malha digital do IBGE (2016). 


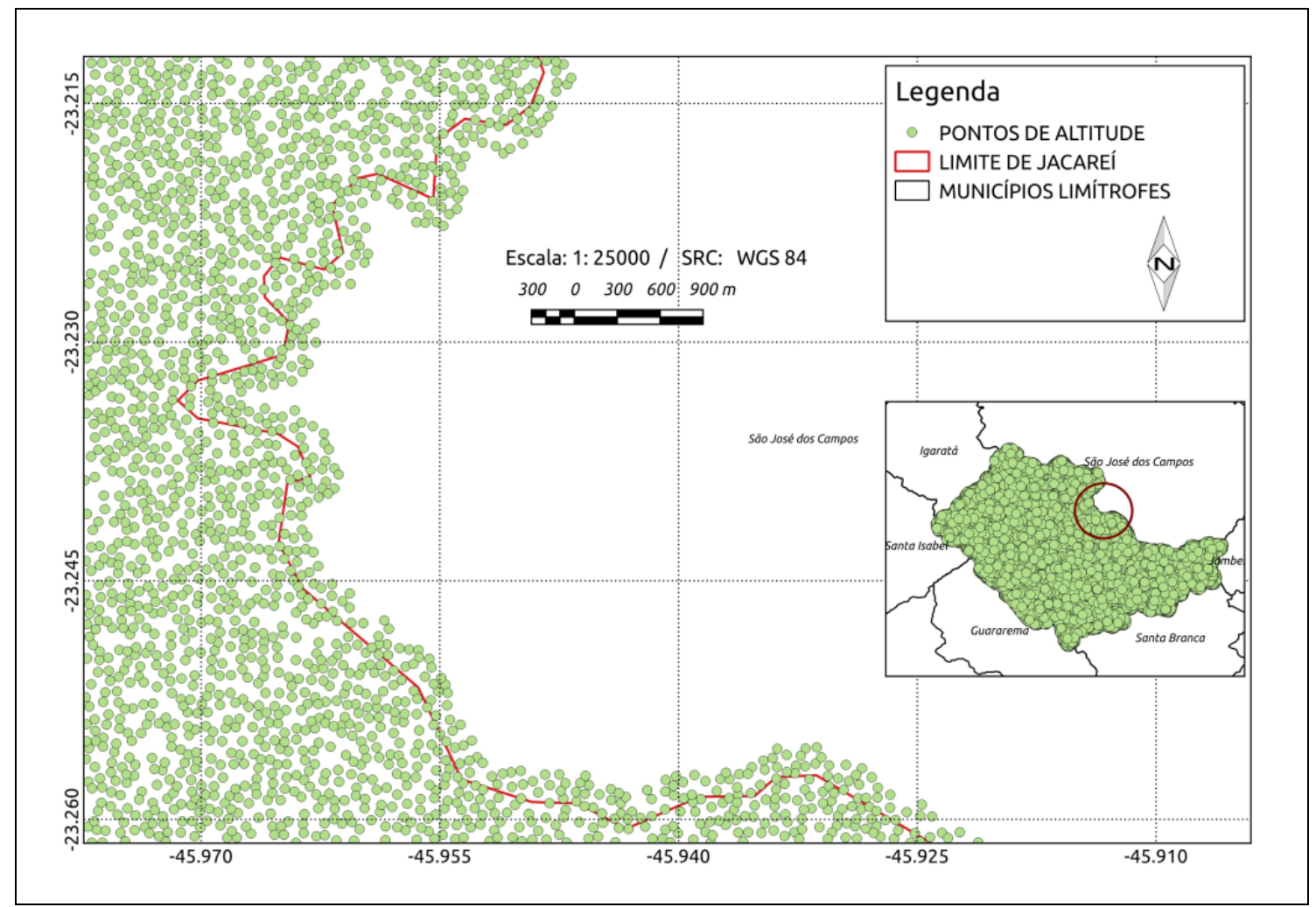

Figura 2. Pontos amostrados em Jacareí (AUTOR, 2019).

\subsection{Método de Triangulação}

Para a representação dos pontos coletados, utilizou-se o método de Delaunay para se fazer a Rede Triangular Irregular. A triangulação por este método pode ser entendida, de acordo com Chew (1989), como a linha reta dual do diagrama de Voronoi (Figura 3). Uma das definições dada pelo autor citado é referente a triangulações irrestritas:

Seja S um conjunto de pontos no plano. Uma triangulação T é uma Triangulação de Delaunay de $\mathrm{S}$ se para cada aresta $\mathrm{E}$ de $\mathrm{T}$ existe um círculo $\mathrm{C}$ com as seguintes propriedades:

1. Os pontos finais da aresta $\mathrm{E}$ estão no limite $\mathrm{E}$ de $\mathrm{C}$.

2. Nenhum outro vértice de $\mathrm{S}$ está no interior de $\mathrm{C}$.

Se não existem quatro pontos de $\mathrm{S}$ que são cocirculares (quatro ou mais pontos no mesmo círculo), então se pode dizer que a triangulação de Delaunay é única. Para a maioria dos casos em que não há uma triangulação de Delaunay única, qualquer delas irão satisfazer as condições necessárias para triangulação.

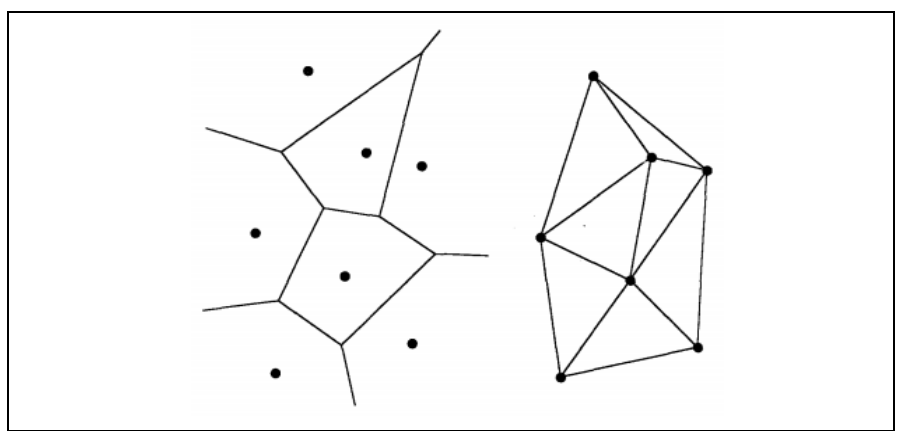

Figura 3. Diagrama de Voronoi e a Triangulação de Delaunay correspondente (CHEW, 1989). 


\section{ISSN $2447-5378$}

\subsection{Perfil Altimétrico e Distância Percorrida}

Para o Instituto Brasileiro de Geografia e Estatística (IBGE, 1999), o perfil que possui relação com o contexto em questão é a representação em termos cartográficos de uma seção vertical da superfície terrestre. De maneira que, inicialmente, precisa-se conhecer as altitudes de um determinado número de pontos e a distância entre eles.

A Figura 4 ilustra a representação de um perfil topográfico de acordo com uma seção traçada entre as curvas de nível.

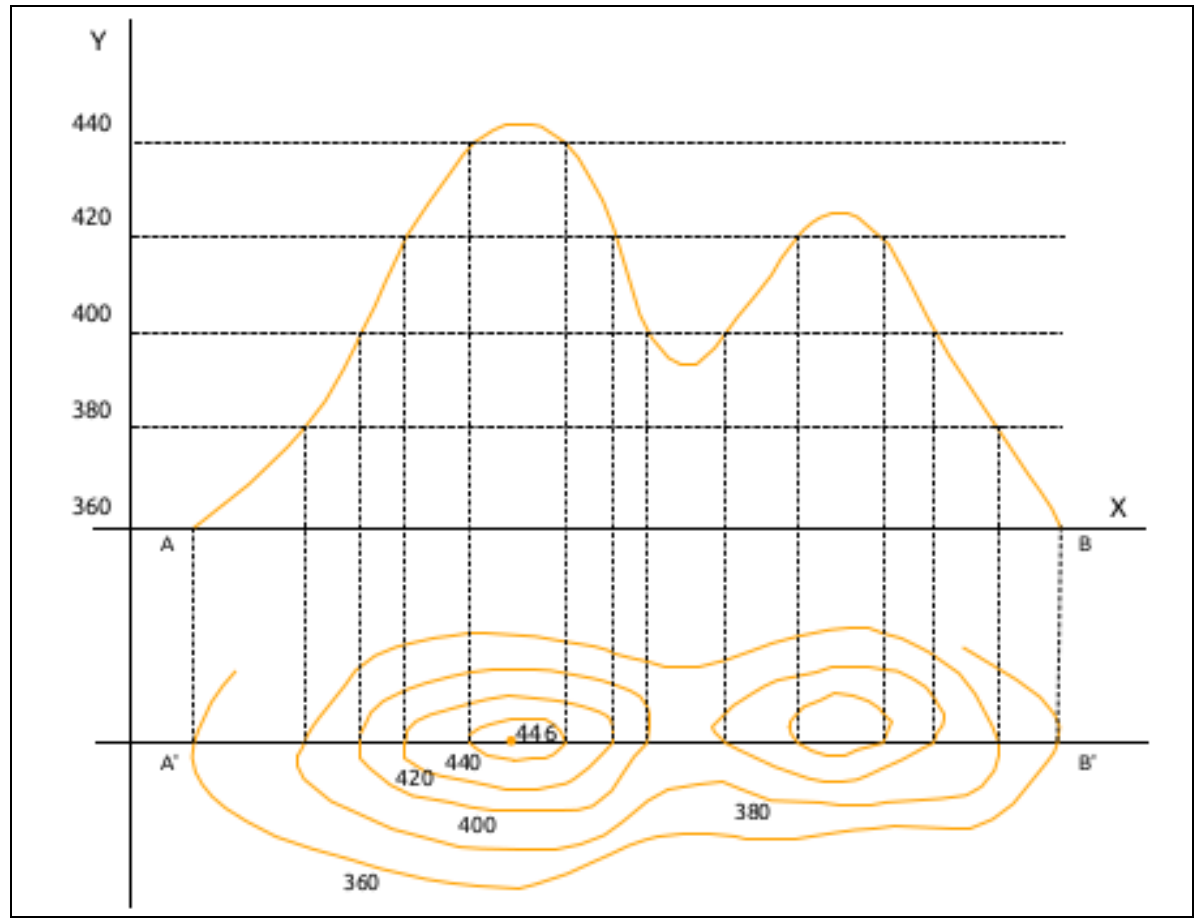

Figura 4. Exemplo de Perfil Topográfico. Adaptado do IBGE (1999).

A fórmula de Haversine fornece basicamente a distância, em radianos, entre dois pontos por meio de longitudes e latitudes em uma esfera (ESENBUĞA et al., 2016). É utilizada pelo Turf e de acordo com Sinnott (1984) existem duas diferentes maneiras de calcular a distância por meio desta fórmula, isto ocorre por conta da relação entre as funções inversas da tangente e do seno, de modo que os resultados serão os mesmos.

É possível ver a seguir parte do pseudocódigo da fórmula de Haversine adaptado de Esenbuğa et al. (2016) por meio das equações 1, 2 e 3:

$$
\begin{aligned}
& a \leftarrow \sin \left(\left(\frac{1}{2} \Phi_{2}-\frac{1}{2} \Phi_{1}\right)^{2}\right)+\sin \left(\left(\frac{1}{2} \lambda_{2}-\frac{1}{2} \lambda_{1}\right)^{2}\right) \cos \left(\Phi_{1}\right) \cos \left(\Phi_{2}\right) \\
& c \leftarrow 2 \text { atan }\left(\frac{\sqrt{a}}{\sqrt{1-a}}\right) \quad \text { ou } \quad c \leftarrow 2 a \sin (\sqrt{a}) \\
& \text { Haversine_Distance } \leftarrow \text { R. } c
\end{aligned}
$$

Onde,

- $\Phi_{1}$ - latitude inicial;

- $\Phi_{2}$ - latitude final; 
- $\lambda_{1}$ - longitude inicial;

- $\lambda_{2}$-longitude final;

- Haversine_Distance - Distância entre dois pontos por meio da fórmula de Haversine;

- $\mathrm{R}$ - é o raio da esfera, sendo que no caso apresentado é o raio médio de acordo com o elipsóide padrão utilizado no WGS 84 (revisão do Sistema Geodésico Mundial - World Geodetic System, de 1984) que é, aproximadamente, $6.371 \mathrm{~km}$ de acordo com a União Internacional de Geodésia e Geofísica - International Union of Geodesy and Geophysics ou IUGG (MISRA; RAMESH, 1989).

\subsection{Tecnologia da Aplicação}

Para a representação dos pontos coletados, utilizou-se o método de Delaunay para se fazer a Rede Triangular Irregular. Os mapas que servem como base da aplicação e possuem serviço gratuito são: Open Street Map (OSM) e Open Topo Map. As linguagens de marcação e formatação são, respectivamente, Html5 e CSS3. Já as linguagens de programação utilizadas foram Javascript e Python em sua versão 3.6.8, junto às suas respectivas bibliotecas (Tabela 1).

Tabela 1. Principais bibliotecas utilizadas.

\begin{tabular}{cc}
\hline Javascript & Python \\
\hline Jquery & Base 64 \\
\hline Leaflet & Flask \\
\hline Plotly & Matplot \\
\hline Turf & Pip \\
\hline React & Urllib \\
\hline
\end{tabular}

Fonte: Autor (2019).

\subsection{Hospedagem}

Utilizou-se um serviço gratuito para o deployment chamado Heroku (HEROKU, 2019) que já hospeda a aplicação nesta sua versão inicial no seguinte endereço: https://app-vt.herokuapp.com. O site hospedeiro usado tem como benefício ser gratuito para pequenas aplicações, além de ter informações de fácil acesso. Assim, um objetivo secundário de conseguir que usuários, possuintes de algum conhecimento em programação, reproduzam para outras cidades o que foi feito para Jacareí pode ser atendido. Existe a probabilidade de isto ocorrer por conta da disponibilização pública do código da aplicação que será dada por intermédio da plataforma chamada GitHub (GITHUB, 2019).

\section{RESULTADOS E DISCUSSÃO}

Os dados em um primeiro momento devem ser carregados pelo usuário, isto acontece após se clicar no botão de carregamento situado do lado inferior direito, em seguida o utilizador escolhe 


\section{ISSN $2447-5378$}

qual município quer carregar para efetuar a modelagem (Figura 5). Após o carregamento efetuado, pode-se fazer as interações com o mapa já com todos os botões apresentados.

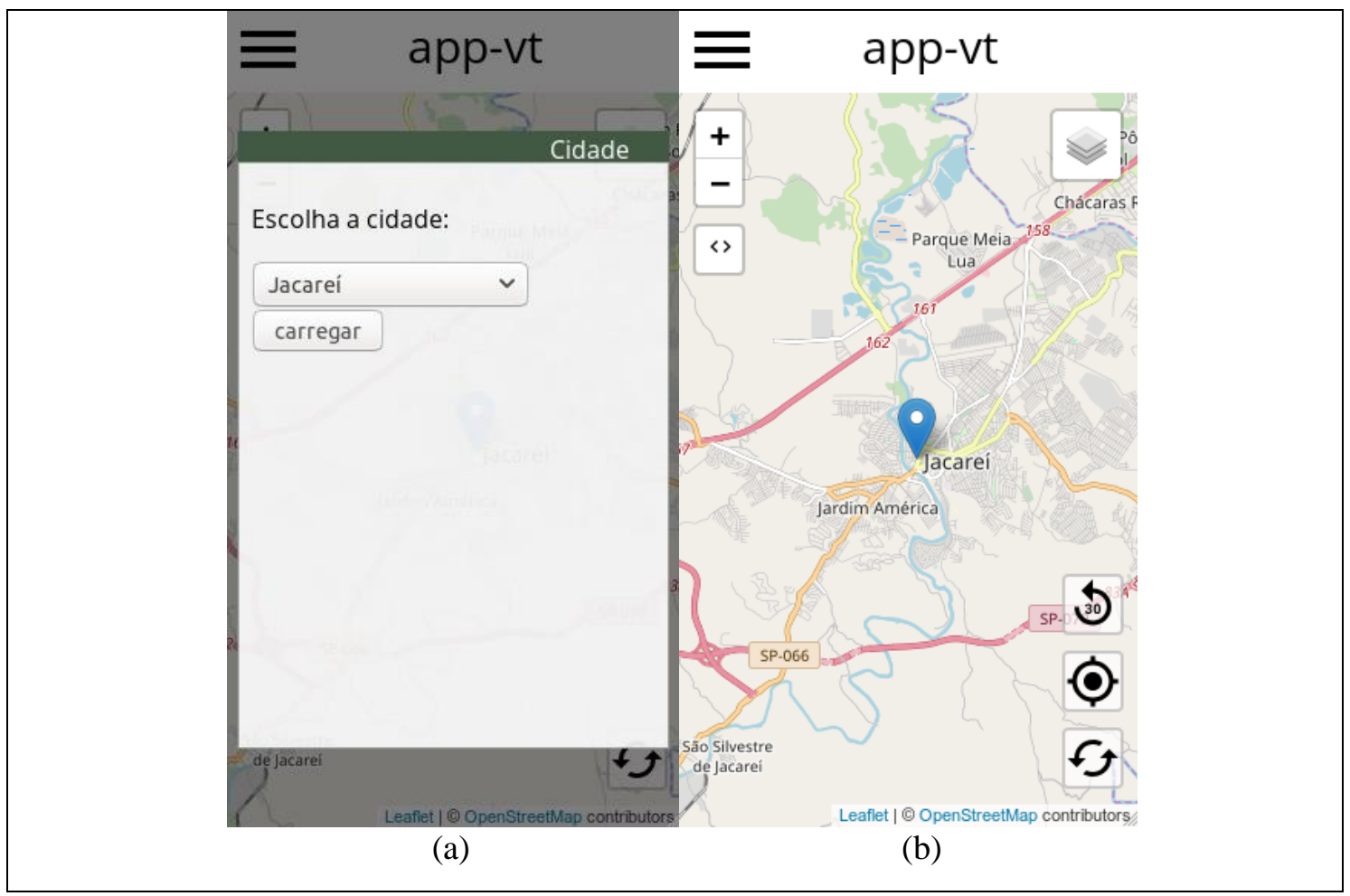

Figura 5. Mostra a escolha da cidade para se carregar os dados em (a) e o carregamento efetuado com os botões apresentados em (b) (AUTOR, 2019).

Ao se clicar no botão que possui simbologia de alvo e depois ir ao primeiro botão do mapa situado do lado superior direito, o usuário terá como resultado o TIN em duas dimensões, necessitando somente selecionar as camadas correspondentes no ícone layers como mostrado na Figura 6.

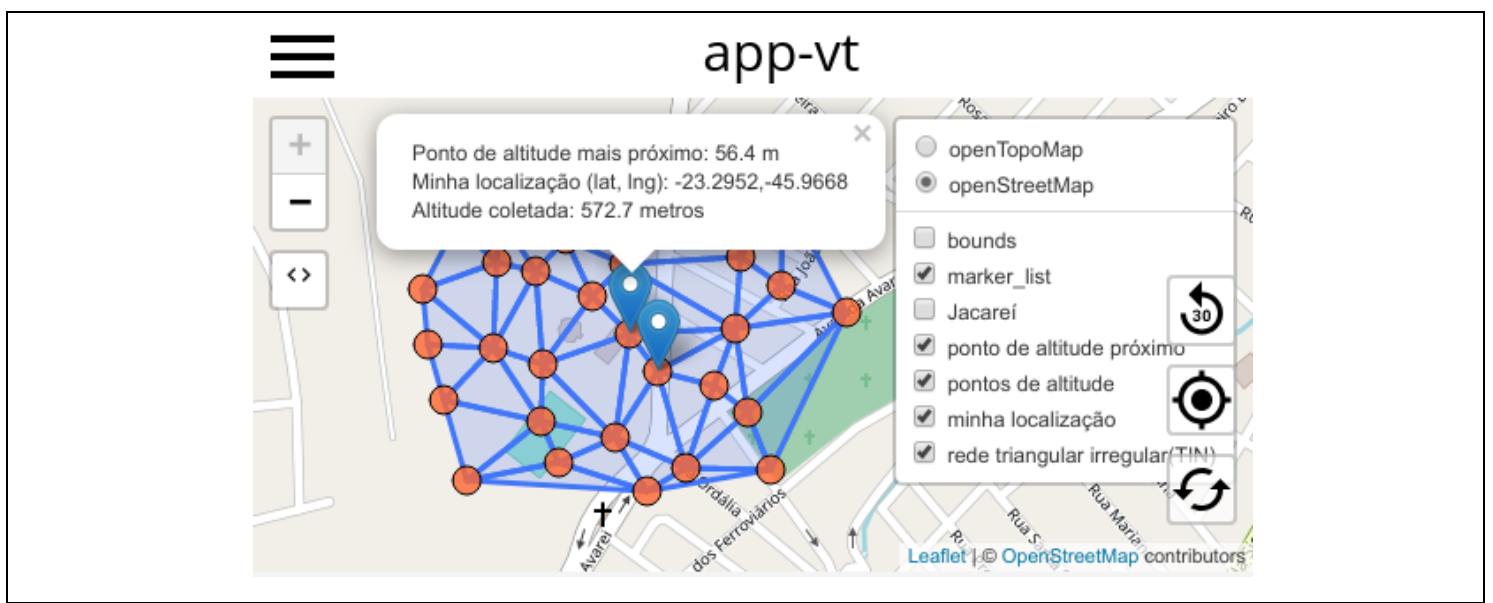

Figura 6. Resultado do TIN (AUTOR, 2019).

Ressalta-se que a seleção da base de dados já mostrada na Figura 6 serviu para gerar o gráfico do TIN em três dimensões que pode ser visualizado na Figura 7. Esta última mostra o resultado em formato GIF (Graphic Interchange Format), todavia este gráfico requer um processamento que pode 


\section{ISSN $2447-5378$}

ser considerado dispendioso por usar várias imagens, já que diferentes pontos de vista são necessários para sua formação. Uma maneira de contornar o problema seria diminuir a qualidade das imagens, ou ainda salvar estas últimas em um formato diferente no futuro para contornar um possível problema de sobrecarga no servidor.

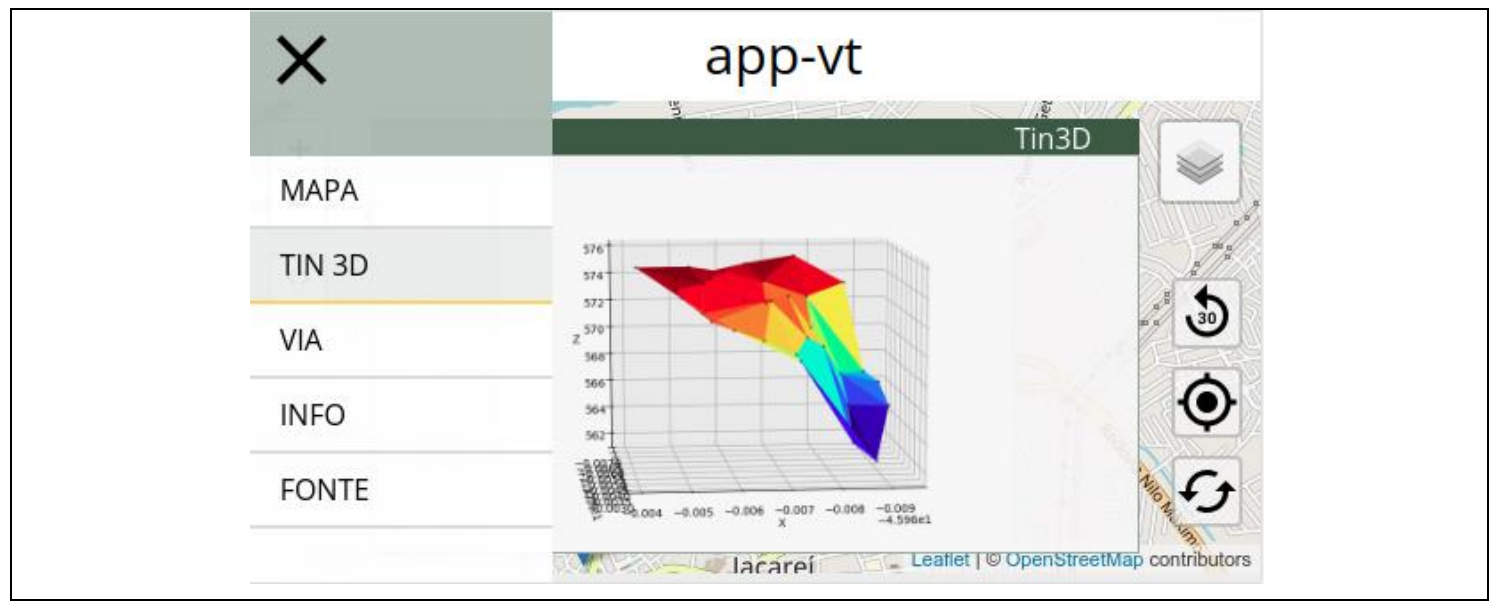

Figura 7. Resultado do TIN 3D (AUTOR, 2019).

O perfil do caminho percorrido também é gerado com o auxílio da base de dados carregada previamente ao se clicar no botão de carregamento e depois no ícone de atualizar a cada 30 segundos, desta forma o resultado pode ser visualizado na aba VIA pelo usuário como mostra a Figura 8 à esquerda. Além disso, pode-se ver à direita (Figura 8) a distância do caminho percorrido entre os pontos de localização ao se clicar no próprio marcador.

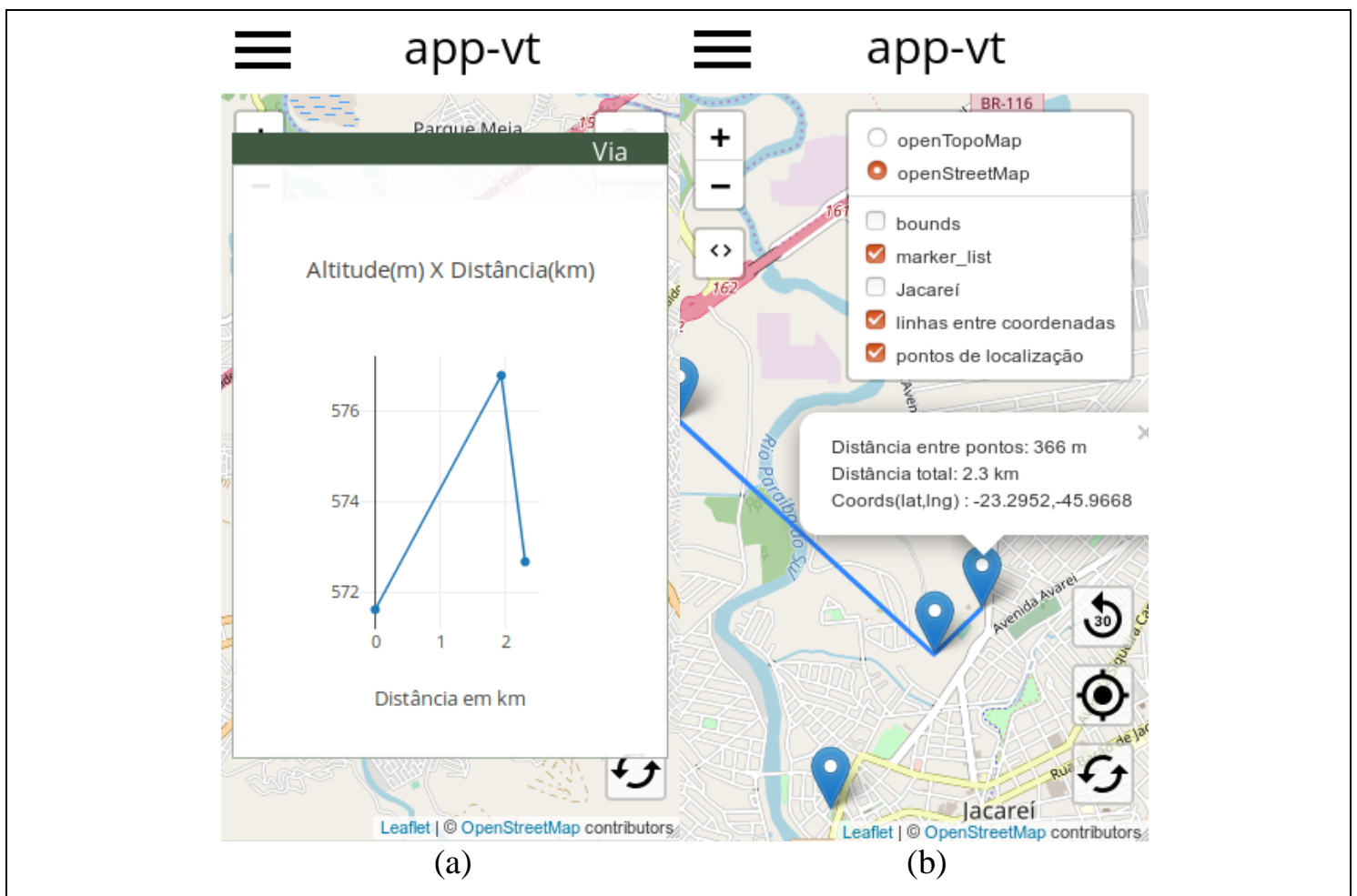

Figura 8. Mostra o gráfico do caminho percorrido em (a) e sua visualização no mapa em (b). (AUTOR, 2019). 


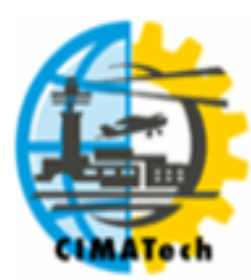

\section{ISSN 2447-5378}

Um ponto que deve ser considerado em relação à questão das medidas de distância (Figura 8) recai sobre a utilização da fórmula de Haversine, esta leva em consideração a curvatura da terra e é usada pela biblioteca Javascript Turf. Contudo, em situações com deslocamento curto, um cálculo em um plano cartesiano poderia ter melhores resultados.

\section{CONCLUSÃO}

Hoje em dia o uso da geolocalização e tecnologias geográficas já é uma ocorrência comum na sociedade e, por isso, é importante a adaptação a esta nova realidade, sendo interessante entender alguns princípios e conceitos inseridos no tema. Neste contexto, aplicações que são utilizadoras de tecnologias geográficas, como a apresentada aqui, podem ter seu valor entre aqueles que desejam conhecer um pouco mais de geoprocessamento, tendo em vista sua importância e aplicabilidade.

Ainda que não seja possível medir a eficácia do mecanismo por meio de opiniões de usuários ou comparações efetivas com outras aplicações, já que é uma primeira versão muito mais próxima a um protótipo e precisa de aprimoramentos antes de se efetuar testes comparativos, há chances futuras de uma expansão para outros tipos de representação de terreno por meio de interpolações e algoritmos não apresentados aqui, conforme ocorrerem modificações e ampliações no código em versões posteriores.

Fato é que, apesar dos problemas possíveis com sobrecarga do servidor ou acurácia insatisfatória em se tratando de medida de curta distância, o primeiro intuito foi atingido, ou seja, o desenvolvimento inicial de uma Aplicação Progressiva Web que visa ajudar os interessados em geotecnologias a compreenderem, de forma simplificada, os fundamentos da geração de um Modelo de Terreno através de sua representação por Rede Triangular Irregular. Apesar da aplicação já estar publicada e em funcionamento, a falta de testes sólidos ainda não possibilita seu uso como ferramenta pedagógica auxiliar por conta da necessidade de melhoramentos no código a serem feitos em futuras versões.

\section{REFERÊNCIAS}

ANA. Agência Nacional de Águas. Sub-bacias Hidrográficas DNAEE. 2018. Disponível em: <http://dadosabertos.ana.gov.br/datasets/10480692111f443bb5a38d9bb156851f_0>. Acesso em: 26 mai. 2019.

ALTER, T. Building Progressive Web Apps: Bringing the Power of Native to the Browser. 1. ed. Sebastopol: O’Reilly Media Inc, 2017.

BHARGAVA, N.; BHARGAVA, R.; TANWAR, P. S. Triangulated Irregular Network Model from Mass Points. International Journal of Advanced Computer Research, v. 3, n. 2, jun. 2013. ISSN (print): 2249-7277 e ISSN (online): 2277-7970. Disponível em: <http://citeseerx.ist.psu.edu/ viewdoc/download?doi=10.1.1.414.3957\&rep=rep1\&type=pdf > . Acesso em: 18 mai. 2019.

CHEW, P. Constrained Delaunay Triangulations. Algorithmica, New York, v. 4, p. 97-108, jun. 1989. Disponível em: <https://doi.org/10.1007/BF01553881>. Acesso em: 20 mai. 2019.

EGG, G. C. Geração de modelos digitais de superfície compostos utilizando imagens do sensor PRISM/ALOS. Dissertação (Mestrado em Engenharia Civil) - Universidade Federal de Viçosa. 


\section{ISSN 2447-5378}

Viçosa, p. 157, 2012. Disponível em: <http://www.locus.ufv.br/bitstream/handle/123456789/ 3783/texto\%20completo.pdf?sequence=1>. Acesso em: 03 dez. 2018.

DUMITRU, P. D.; PLOPEANU, M.; BADEA, D. Comparative study regarding the methods of interpolation. Recent advances in geodesy and Geomatics engineering. 2013. ISBN: 978-960474-335-3.

ESENBUĞA, Ö. G. et al. Comparison of principal geodetic distance calculation methods for automated province assignment in Turkey. In: International Multidisciplinary Scientific GeoConference Surveying Geology \& mining Ecology Management (SGEM), 16., 2016. SGEM 2016 Conference Proceedings, Book 2, v. 2, p.141-148. ISBN 978-619-7105-59-9/ISSN 1314-2704. Disponível em: <https://doi.org/10.5593/SGEM2016/B22/S09.019>. Acesso em: 25 mai. 2019.

FELGUEIRAS, C. A. Análises sobre modelos digitais de terreno em ambiente de sistemas de informações geográficas. Divisão de Processamento de Imagens do Instituto Nacional de Pesquisas Espaciais. 1999. Disponível em: <https://www.researchgate.net/publication/47 445961_Analise_sobre_modelos_digitais_de_terreno_em_ambiente_de_sistemas_de_informacoes_ geograficas>. Acesso em: 02 dez. 2018.

GITHUB. 2019. Disponível em: <https://github.com/>. Acesso em: 10 jun. 2019.

HEROKU. 2019. Disponível em: <https://www.heroku.com/>. Acesso em: 10 jun. 2019.

IBGE. Instituto Brasileiro de Geografia e Estatística. Malhas Digitais. 2016. Disponível em: <https://mapas.ibge.gov.br/bases-e-referenciais/bases-cartograficas/malhas-digitais.html>. Acesso em: 26 mai. 2019.

Noções Básicas de Cartografia: III - Elementos de representação. Manuais Técnicos em Geociências, n. 8. p. 69-89. 1999. Disponível em: <https://biblioteca.ibge.gov.br/ visualizacao/monografias/GEBIS\%20-\%20RJ/ManuaisdeGeociencias/Nocoes\%20basicas\%20de\%2 0cartografia.pdf $>$. Acesso em: 05 dez. 2018.

. Panoramas. 2018. Disponível em: <https://cidades.ibge.gov.br/brasil/sp/jacarei/panorama >. Acesso em: 05 dez. 2018.

JACOBI, P. Educação ambiental, cidadania e sustentabilidade. Cadernos de pesquisa, n. 118, 2003. Disponível em: 〈http://www.scielo.br/pdf/cp/n118/16834.pdf>. Acesso em: 01 dez. 2018.

LANDIM, P. M. B. Sobre Geoestatística e mapas. Terræ Didatica, v. 2, p. 19-33, 2006. Disponível em: <http://www.ige.unicamp.br/terraedidatica/>. Acesso em: 02 dez. 2018.

LI, Z.; ZHU, Q.; GOLD, C. Digital terrain modeling: principles and methodology. Boca Raton, Florida: CRC Press, 2005.

MISRA, R. P; RAMESH, A. Fundamentals of Cartography. Revised \& Enlarged ed. New Delhi: Concept Publish Company, 1989. ISBN 81-7022-222-2.

MORAN, J. M. Como utilizar a Internet na educação. Ciência da informação, Brasília, v. 26, n.2, 1997. Disponível em: <https://dx.doi.org/10.1590/S0100-19651997000200006>. Acesso em: 06 dez. 2018. 


\section{ISSN 2447-5378}

ROSSUM, G. V; DRAKE JR., F. An Introduction to Python. 1. ed. United Kingdom: Network Theory Ltd, 2011.

SINNOTT, R. W. Virtues of the Haversine. Cambridge, Sky \& Telescope, v. 68, n. 2, p. 159, ago. 1984.

TESKE, R.; GIASSON, E.; BAGATINI, T. Comparação do uso de modelos digitais de elevação em mapeamento digital de solos em Dois Irmãos, RS, Brasil. Viçosa, Revista Brasileira de Ciência do Solo, v. 38, n. 5, setembro/out. 2014. ISSN 1806-9657. Disponível em: <http://dx.doi.org/ 10.1590/S0100-06832014000500002>. Acesso em: 25 mai. 2019.

TOPODATA MAPA ÍNDICE. 2019. Disponível em: <https://www.webmapit.com.br/inpe/ topodata/>. 2019. Acesso em: 26 mai. 2019.

UNITED STATES GEOLOGICAL SURVEY. SRTM data have been enhanced to fill areas of missing data to provide digital elevation data with a resolution of 1 arc-second for global coverage. 2018. Disponível em: <https://www.usgs.gov/centers/eros/science/usgs-eros-archivedigital-elevation-shuttle-radar-topography-mission-srtm-1-arc?qt-science_center_objects=0\#qtscience_center_objects>. Acesso em: 18 mai. 2019.

. What is the difference between a DEM and a DSM? 2019. Disponível em: <https:// www.usgs.gov/faqs/what-difference-between-a-dem-and-a-dsm>. Acesso em: 18 mai. 2019. 